\title{
Is Only Reduction in CD4 Count Responsible for Secondary Infections Seen in the HIV Patients?
}

\author{
Rajeev Shah ${ }^{1}$ and Aarjav Shah ${ }^{2}$ \\ ${ }^{1}$ Professor and Head, Microbiology Department, PMCH, Pacific University, Udaipur, India \\ ${ }^{2}$ Sheenathaji Clinic, Mumbai, India \\ *Corresponding Author: Rajeev Shah, Professor and Head, Microbiology Department, PMCH, Pacific University, Udaipur, India.
}

Received: July 22, 2019; Published: September 01, 2019

DOI: 10.31080/ASMI.2019.02.0359

Keywords: Tuberculosis; HIV; CD4 Count; ART; CD8 Count

Background: The significant mean CD4 count fall has been observed both in HIV positive patients as well as even with the patients of severe tuberculosis without HIV infections.. But it has been observed that generally, the patients with tuberculosis do not seems to have secondary or opportunistic microbial infections, while in contrast, the HIV patients with same mean CD4 count suffer from plenty of opportunistic or secondary infections.

Aim: Emphasizing the pivotal role of CD4 count in TB/HIV patients in maintaining their immune system effective (by maintaining CD4 count) and thus decreasing MDR/XDR, morbidity and mortality among these patients. Calculating average mean CD4 count for Indian scenario in cART era. Discussing and suggesting new scope of treating HIV patients for prevention of secondary infections.

Material and Methods: All the 961 HIV infected patients early morning sputa were screened for AFB and few of the samples were even cultured on LJ medium. All patients' CD4 count were also evaluated by flow cytomerty method within one week of sputa collection. Seven other published work of HIV/TB patients were analyzed in relation to CD4 count. Moreover other five published research on CD4 in TB+ve/HIV-ve patients were also discussed in this article.

Results: Out of 961 patients with HIV/RTI, 308(32.06\%) found positive for tuberculosis with mean CD4 count found to be 198.5 and 105.9 cells/ $\mu$ l for pulmonary TB and for extra-pulmonary TB respectively in present study. The average mean CD4 count from seven research studies from India were found to be 169.75 and 145.3 cells/ $\mu$ f for pulmonary and extra-pulmonary TB respectively, in TB/HIV co-infected patients on cART. Brenda., et al. (1997) and other four found that in advanced/sever TB but HIV-negative patients mean CD4 count found to be $341+116$. It means in severe tuberculosis patients CD4 count may reduce up to 198 cells $/ \mu \mathrm{l}$ but in TB patients, But the difference between HIV and TB patients found by researchers was the CD4:CD8 ratio which always almost maintained in TB patients only but not in HIV patients. Even some researchers like MA Hauman, Fiske CT., et al. (2015) could not find increased intracellular bacterial infections (ICBIs) in only TB patients (HIV-ve).

Conclusion: HAART and ATT both are equally important in maintaining immune system (maintaining CD4 count) of TB/HIV co-infected patients. In India, clinician should suspect more for TB at around mean CD4 count of 169.75 even if found negative by AFB staining, they required to confirm TB by culture on LJ medium, PCR, gene expert or by any other Latest technique in HIV-positive patients. It is not only reduced CD4 count responsible for secondary infections seen in HIV patients but it might be spoiled CD4:CD8 ratio, or in other world increased CD8 cells in comparison with CD4 cells might be responsible for secondary infections seen in HIV patients, to confirm this further research is required. In severe TB with CD4 count even below 200 cells/ $\mu \mathrm{l}$, secondary infections are usually not seen, so which other factors are there which prevent secondary infections in TB patients even with CD4 count below 200 cells/ $\mu \mathrm{l}$. One difference between TB and HIV patients seen is the ratio of CD4:CD8 cell count, which is always seems to be normal in only patients of Tuberculosis alone, in contrast to this in HIV patients the CD4:CD8 ratio always found abnormal. So if we maintain CD4:CD8 ratio in HIV patients, by giving anti-antibodies to CD8 appropriately or by any other methods, it should theoretically reduce/stop secondary or opportunistic infections in HIV patients also. Further intensive practical research is required to find out new scope to reduce/stop secondary infections in HIV patients in this direction.

cART $=$ Combined Antiretroviral Treatment

ATT $=$ Anti Tubercle Treatment

Volume 2 Issue 10 October 2019

(C) All rights are reserved by Rajeev Shah and Aarjav

Shah. 